\title{
Transitive preferences in multi-member households
}

\author{
Bart Smeulders* Laurens Cherchye, Bram De Rock ${ }^{\ddagger}$ \\ Frits C.R. Spieksma ${ }^{\S}$ and Fabrice Talla Nobibon $₫$
}

July 2, 2014

\begin{abstract}
The classical result of [14] shows that, for single-member households, the Weak Axiom of Revealed Preferences (WARP) and the Strong Axiom of Revealed Preferences (SARP) are equivalent when there are only 2 goods. Because SARP extends WARP by requiring transitive preferences in addition, this means that transitivity of preferences need not be tested in the case of two goods. We consider the extension of this result towards $L$-member households, for which we introduce the concepts $L$-WARP and $L$-SARP. For a general setting, we demonstrate that $L$-WARP and $L$-SARP are not equivalent if there are at least $L+1$ goods, which means that transitivity of individual preferences is testable. However, we can also show that $L$-WARP and $L$-SARP do become equivalent for the restricted "labor supply" setting where we exclusively assign a single good to each different household member, i.e. $L$ (out of $L+1$ ) goods are exclusive.
\end{abstract}

Keywords: Weak Axiom of Revealed Preferences; Strong Axiom of Revealed preferences; multi-member households; transitivity.

JEL Classification: D11, D12, D13, C14.

\section{Introduction}

We study consumption decisions (including labor supply decisions) of multi-member households. By now, it is well established that the collective model of [10] is both conceptually and empirically attractive for analyzing consumption behavior (see, for example, [22] for an overview of the relevant literature). This collective model assumes that the different household members are endowed with individual preferences defined over privately and publicly consumed goods (inside the household). These members enter into a decision process of

\footnotetext{
*ORSTAT, University of Leuven.

${ }^{\dagger}$ Center for Economic Studies, University of Leuven. Laurens Cherchye gratefully acknowledges financial support from the Research Fund K.U.Leuven through the grant STRT1/08/004.

${ }^{\ddagger}$ Corresponding author, ECARES, Université Libre de Bruxelles, Avenue F.D. Roosevelt 50, CP 114-04, B-1050 Brussels, Belgium, e-mail: bderock@ulb.ac.be. Bram De Rock gratefully acknowledges the European Research Council (ERC) for his Starting Grant.

$\S$ ORSTAT, University of Leuven. Frits Spieksma's research has been partially funded by the Interuniversity Attraction Poles Programme initiated by the Belgian Science Policy Office.

๑ORSTAT, University of Leuven.
} 
which the outcome is assumed to obtain a Pareto optimal allocation (of the aggregate household budget). In what follows, we say that multi-person household behavior is collectively rational if it is consistent with the collective model, see Section 2 for a formal statement.

In the tradition of [1] and [20], we are interested in the revealed preference characterization of collective models. Such a revealed preference characterization does not rely on any functional specification regarding the household consumption process, and starts directly from the observed finite set of prices and quantities. [20] introduced the revealed preference axioms that summarize the empirical implications of theoretical consumption models for single-member households, while [7] provided a revealed preference characterization of collective models for multi-member households. ${ }^{1}$

Our following analysis focuses on two popular revealed preference axioms: the Weak $A x$ iom of Revealed Preferences (WARP) introduced by [15], and the Strong Axiom of Revealed Preferences (SARP) introduced by [12]. For single-person households, these axioms summarize the testable implications of rational (i.e. utility maximizing) consumption behavior. Essentially, SARP extends WARP by requiring preferences to be transitive (see, again, Section 2 for formal definitions). In this respect, [14]'s classical result shows that WARP and SARP are empirically equivalent in a setting with 2 goods. In other words, transitivity has no empirical bite if the analysis includes only 2 goods. Because WARP is generally easier to test than SARP, this result can considerably facilitate the computational burden of the empirical analysis. ${ }^{2}$ Moreover, following [19], the validity of transitive preferences has been put in question in the literature on behavioral economics. ${ }^{3}$ Importantly, the result of [14] implies that such considerations cannot be meaningfully investigated in a setting with not more than two goods.

In what follows, we investigate the possibility to extend [14]'s result towards multimember households. Therefore, we define the concepts of $L$-WARP and $L$-SARP, which capture the testable implications of collectively rational (i.e. Pareto efficient) consumption behavior in the case of $L$ household members. In a first instance, we assume a general setting in which we only observe the aggregate household consumption, i.e. no information is available on the intrahousehold allocation of the private goods. First, we show that $L$-WARP and $L$-SARP are empirically vacuous (i.e. non-rejectable) if there are not more than $L$ goods. Next, and more importantly, we show that WARP and SARP are not equivalent if there are at least $L+1$ goods. Thus, [14]'s conclusion does not generalize for $L$-member households (even with as few as four observations). Therefore, transitivity of individual preferences is a testable requirement even if there are only $L+1$ goods for $L$ household members.

We also study a more restricted setting where we exclusively assign a single good to each different household member, i.e. $L$ (out of $L+1$ ) goods are exclusive. Thus, in contrast to the general setting, this restricted setting assumes that we observe the intrahousehold allocation of $L$ goods. We call this a "labor supply" setting as it formally coincides with [10]'s original labor supply model, in which each household member exclusively consumes his/her (observed) leisure while the remaining consumption is captured by a Hicksian aggregate (that is to be shared among the household members). Interestingly, we can show that $L$-WARP and $L$-SARP do become equivalent under these empirical conditions (with $L+1$ goods). Thus,

\footnotetext{
${ }^{1}$ See also [13], [21], [5], [6] and [9] for more discussion.

${ }^{2}$ See [17] for a recent study on the computational complexity of testing WARP and SARP.

${ }^{3}$ See, for example, [3] for a recent review.
} 
the empirical analysis need not explicitly account for transitivity, which can substantially alleviate the computational burden in practical applications.

As a final remark, we note that there is close relation between our household consumption models and general equilibrium models. ${ }^{4}$ In this respect, the classic Sonnenschein-MantelDebreu result also states that general equilibrium models do not generate testable implications (for the excess demand function) in an economy with at least as many agents as commodities. In an important contribution, [2] circumvent this negative result by developing revealed preference characterizations of general equilibrium behavior that are based on the equilibrium manifold (and not the excess demand function). ${ }^{5}$ More recently, [8] extend these results and present revealed preference characterizations of general equilibrium models that are closely related to the collective models that we consider in this paper. This shows that our following results can actually also be relevant for general equilibrium analysis.

The rest of the paper unfolds as follows. In Section 2 we introduce the collective model and the corresponding revealed preference axioms. In Section 3 we investigate the equivalence between $L$-WARP and $L$-SARP for both the general setting and the restricted labor supply setting. Section 4 concludes. The Appendix contains the proofs of our main results.

\section{Revealed preference characterization of collective household behavior}

Notation and definitions. We consider an $L$-member household that consumes $N$ private goods and $K$ public goods (with $L, N, K \in \mathbb{N}_{0}$ ). The vector $\mathbf{q} \in \mathbb{R}_{+}^{N}$ represents the quantities that are privately consumed by the household, and $\mathbf{p} \in \mathbb{R}_{+}^{N}$ stands for the corresponding price vector. Similarly, the vector $\mathbf{Q} \in \mathbb{R}_{+}^{K}$ represents the publicly consumed quantities, and $\mathbf{P} \in \mathbb{R}_{+}^{K}$ gives the price vector for the public goods. Next, the vector $\mathbf{q}^{\ell} \in \mathbb{R}_{+}^{N}$ contains the privately consumed quantities for each individual member $\ell$, with $\sum_{\ell=1}^{L} \mathbf{q}^{\ell}=\mathbf{q}$. The collective model of household consumption explicitly recognizes the individual preferences of the household members. These preferences may depend on the private quantities, the public quantities, or both. Throughout, we assume that preferences of member $\ell$ can be represented by a well-behaved (i.e. continuous, positive monotonic and concave) utility function $U^{\ell}\left(\mathbf{q}^{\ell}, \mathbf{Q}\right), \ell=1, \ldots, L$.

Our analysis starts from the data set $S=\left\{\left(\mathbf{p}_{t}, \mathbf{P}_{t} ; \mathbf{q}_{t}, \mathbf{Q}_{t}\right), t=1, \ldots, T\right\}$, which contains $T$ household choices that are characterized by prices $\mathbf{p}_{t}, \mathbf{P}_{t}$ and quantities $\mathbf{q}_{t}, \mathbf{Q}_{t}$. In our general setting, we do not know which quantities are privately consumed by which member, i.e. $\mathbf{q}_{t}^{\ell}$ is unobserved. Therefore, we need to introduce (unobserved) feasible personalized quantities that comply with the (observed) aggregate quantities $\mathbf{q}_{t}$. That is, we consider all possible decompositions $\mathbf{q}_{t}^{\ell} \in \mathbb{R}_{+}^{N}$ that satisfy $\sum_{\ell=1}^{L} \mathbf{q}_{t}^{\ell}=\mathbf{q}_{t}$. In what follows, our main focus will be on this general setting. However, as indicated in the Introduction, we will also consider a

\footnotetext{
${ }^{4}$ See, e.g., [11] for an in-depth discussion of the link between collective models and general equilibrium models.

${ }^{5}$ See, e.g., [4] for an extensive overview of the literature on empirical restrictions of general equilibrium models.
} 
restricted (labor supply) setting that is characterized by exclusive goods. An exclusive good is a private good that is exclusively consumed by a given member. Evidently, this setting implies extra information on $\mathbf{q}_{t}^{\ell}$.

A collective rationalization of a set of observations $S$ requires the existence of memberspecific utility functions for which each observed quantity bundle can be characterized as Pareto efficient. The following definition provides a formal statement. ${ }^{6}$

Definition 1. Let $S=\left\{\left(\mathbf{p}_{t}, \mathbf{P}_{t} ; \mathbf{q}_{t}, \mathbf{Q}_{t}\right), t=1, \ldots, T\right\}$ be a set of observations. Then, the utility functions $U^{1}, \ldots, U^{L}$ provide a collective rationalization of $S$ if, for each observation $t$, there exist feasible personalized quantities $\mathbf{q}_{t}^{\ell}$ such that:

1. $\sum_{\ell=1}^{L} \mathbf{q}_{t}^{\ell}=\mathbf{q}_{t}$, and

2. for all possible quantities $\mathbf{z}^{\ell} \in \mathbb{R}_{+}^{N}, \mathbf{Z} \in \mathbb{R}_{+}^{K}$ with $\sum_{\ell=1}^{L} \mathbf{p}_{t} \mathbf{q}_{t}^{\ell}+\mathbf{P}_{t} \mathbf{Q}_{t} \geq \sum_{\ell=1}^{L} \mathbf{p}_{t} \mathbf{z}^{\ell}+\mathbf{P}_{t} \mathbf{Z}$, we have that if $U^{\ell}\left(\mathbf{z}^{\ell}, \mathbf{Z}\right)>U^{\ell}\left(\mathbf{q}_{t}^{\ell}, \mathbf{Q}_{t}\right)$ then there is some member $m$ for which $U^{m}\left(\mathbf{z}^{m}, \mathbf{Z}\right)<$ $U^{m}\left(\mathbf{q}_{t}^{m}, \mathbf{Q}_{t}\right)$.

Revealed preference characterization. Our revealed preference characterizations of collectively rational behavior make use of the concepts $L$-WARP and $L$-SARP, which provide multi-member extensions of the WARP and SARP concepts that apply to single-member households. To formally define $L$-WARP and $L$-SARP, we need the concept of feasible personalized prices. These are prices $\mathbf{P}^{\ell} \in \mathbb{R}_{+}^{K}$ such that $\sum_{\ell=1}^{L} \mathbf{P}^{\ell}=\mathbf{P}$. Intuitively, these personalized prices capture the fractions of the household prices for the public goods that are borne by the individual members $\ell$. Given the Pareto efficiency assumption that underlies the collective consumption model, these prices can also be interpreted as Lindahl prices. We refer to [7] for a detailed discussion.

Assume that we observe a data set $S=\left\{\left(\mathbf{p}_{t}, \mathbf{P}_{t} ; \mathbf{q}_{t}, \mathbf{Q}_{t}\right), t=1, \ldots, T\right\}$, and consider a given specification of feasible personalized quantities $\mathbf{q}_{t}^{\ell}$ and prices $\mathbf{P}_{t}^{\ell}$. Then, for household member $\ell$, we say that the consumption allocation $s$ is directly revealed preferred over another allocation $t$ (denoted $s R_{0}^{\ell} t$ ) if $\mathbf{p}_{s} \mathbf{q}_{s}^{\ell}+\mathbf{P}_{s}^{\ell} \mathbf{Q}_{s} \geq \mathbf{p}_{s} \mathbf{q}_{t}^{\ell}+\mathbf{P}_{s}^{\ell} \mathbf{Q}_{t}$. The transitive closure of this relation is denoted by $R^{\ell}$. Essentially, the relation $R^{\ell}$ extends $R_{0}^{\ell}$ by exploiting transitivity of individual preferences. We can now define our concepts $L$-WARP and $L$-SARP.

Definition 2. Let $S=\left\{\left(\mathbf{p}_{t}, \mathbf{P}_{t} ; \mathbf{q}_{t}, \mathbf{Q}_{t}\right), t=1, \ldots, T\right\}$ be a set of observations.

1. $S$ satisfies $L$-WARP if and only if there exist for all $\ell=1, \ldots, L$ feasible personalized quantities $\mathbf{q}_{t}^{\ell}$ and feasible personalized prices $\mathbf{P}_{t}^{\ell}$, such that for all pairs of observations $s, t=1, \ldots, T, t R_{0}^{\ell} s$ implies either $\mathbf{p}_{s} \mathbf{q}_{s}^{\ell}+\mathbf{P}_{s}^{\ell} \mathbf{Q}_{s}<\mathbf{p}_{s} \mathbf{q}_{t}^{\ell}+\mathbf{P}_{s}^{\ell} \mathbf{Q}_{t}$ or $\left(\mathbf{q}_{t}^{\ell}=\mathbf{q}_{s}^{\ell}\right.$ and $\left.\mathbf{Q}_{t}=\mathbf{Q}_{s}\right)$.

\footnotetext{
${ }^{6}$ Given that we assume Pareto optimality, we note that condition 2 of Definition 1 can also be reformulated by making use of the second welfare theorem. That is, there needs to exist a distribution of the household income (say $\left.y_{t}^{1}, \ldots, y_{t}^{L}\right)$ such that for each member $\ell\left(\mathbf{q}_{t}^{\ell}, \mathbf{Q}_{t}\right)$ is maximizing $U^{\ell}$ subject to the budget constraint $\mathbf{p}_{t} \mathbf{q}_{t}^{\ell}+\mathbf{P}_{t}^{\ell} \mathbf{Q}_{t} \leq y_{t}^{\ell}$.
} 
2. $S$ satisfies $L$-SARP if and only if there exist for all $\ell=1, \ldots, L$ feasible personalized quantities $\mathbf{q}_{t}^{\ell}$ and feasible personalized prices $\mathbf{P}_{t}^{\ell}$, such that for all pairs of observations $s, t=1, \ldots, T, t R^{\ell} s$ implies either $\mathbf{p}_{s} \mathbf{q}_{s}^{\ell}+\mathbf{P}_{s}^{\ell} \mathbf{Q}_{s}<\mathbf{p}_{s} \mathbf{q}_{t}^{\ell}+\mathbf{P}_{s}^{\ell} \mathbf{Q}_{t}$ or $\left(\mathbf{q}_{t}^{\ell}=\mathbf{q}_{s}^{\ell}\right.$ and $\mathbf{Q}_{t}=\mathbf{Q}_{s}$.

If $L=1$, Definition 2 reduces to the standard definition of WARP and SARP in [20]. When $L \geq 2$, then this definition states that, for the given specification of feasible personalized quantities and prices, $S$ satisfies $L$-WARP if and only if, for each member $\ell$, the feasible personalized prices and quantities satisfy WARP. A directly similar interpretation applies to $L$-SARP, except that this concept also accounts for (indirect) revealed preference relations that are induced by transitivity.

As discussed extensively in [20] and [21], WARP defines a necessary condition for the existence of a well-behaved utility function for single-member households. In general, however, WARP is not sufficient because it does not impose transitivity. By contrast, SARP defines a necessary as well as a sufficient condition. These insights extend to the multi-member setting that we consider here. In particular, the results of [7] are easily adapted to show that there exist utility functions that provide a collective rationalization of $S$ if and only if at least one specification of feasible personalized quantities and feasible personalized prices satisfies $L$-SARP. Again, $L$-WARP provides a corresponding necessary condition by not requiring transitivity of the individual preferences. For results on the computational complexity of testing WARP in various settings we refer to [16].

\section{Is $L$-WARP equivalent to $L$-SARP?}

In this section, we compare the testable implications of $L$-WARP and $L$-SARP. We start by showing that $L$-WARP and $L$-SARP are empirically vacuous conditions if there are no more than $L$ goods. Subsequently, we demonstrate that, for $L+1$ goods (or more), the equivalence between $L$-WARP and $L$-SARP breaks down for the general setting (without exclusive goods). Finally, we also show that $L$-WARP and $L$-SARP do become equivalent for the restricted labor supply setting (where $L$ out of the $L+1$ goods are exclusive).

At most $L$ goods. Before presenting our results, let us first recall that for single-member households WARP and SARP are idle conditions if there is only a single good. Indeed, in that case $t R_{0}^{l} s$ is equivalent to $q_{t} \geq q_{s}$ (with $q_{t}, q_{s} \in \mathbb{R}_{+}$and $s, t=1, \ldots, T$ ), which implies that we can never reject either WARP or SARP. This non-testability result can be extended to $L$-WARP and $L$-SARP, as follows. The proofs of all our results can be found in the Appendix.

Lemma 1. Let $S=\left\{\left(\mathbf{p}_{t}, \mathbf{P}_{t} ; \mathbf{q}_{t}, \mathbf{Q}_{t}\right), t=1, \ldots, T\right\}$ be a set of observations. Then $L$-WARP and $L$-SARP are vacuous conditions as soon as $L \geq N+K$.

Lemma 1 implies that we can only meaningfully check consistency with $L$-WARP and $L$-SARP if the number of goods $N+K$ is strictly larger than $L .^{7}$ Next, it is possible to generalize Example 1 of [5] to show that both $L$-WARP and $L$-SARP can be rejected as soon

\footnotetext{
${ }^{7}$ We note that [18] obtained a similar result in a general equilibrium context with only private goods.
} 
as there are $L+1$ goods. Given this, a natural next question is whether $L$-WARP and $L$-SARP are equivalent for $L+1$ goods. This would generalize [14]'s result (which shows equivalence for $L=1$ ) towards $L \geq 2$.

$L$-WARP and $L$-SARP are not equivalent. For the general setting, the answer to this equivalence question is negative. We can show this for $L=2$ by means of the data set in Example 1.

Example 1. Let us consider a data set consisting of the four observations presented in Table 1 .

\begin{tabular}{|l|l|}
\hline $\mathfrak{q}_{1}=(5,0,0)$ & $\mathfrak{p}_{1}=(7.5,0.5,0.5)$ \\
$\mathfrak{q}_{2}=(0,5,0)$ & $\mathfrak{p}_{2}=(1,2,0.9)$ \\
$\mathfrak{q}_{3}=(0,0,5)$ & $\mathfrak{p}_{3}=(0.2,2.02,2)$ \\
$\mathfrak{q}_{4}=(4,3,1)$ & $\mathfrak{p}_{4}=(1,1,5)$ \\
\hline
\end{tabular}

Table 1: Example data set.

We use this data set to consider two cases. In the "private case" there is only private consumption, i.e. $\mathbf{q}_{t}=\mathfrak{q}_{t}$ and $\mathbf{Q}_{t}=0$ for $t=1,2,3,4$. By contrast, in the "public case" there is only public consumption, i.e. $\mathbf{Q}_{t}=\mathfrak{q}_{t}$ and $\mathbf{q}_{t}=0$ for $t=1,2,3,4$.

For this specific data set we obtain the following result.

Lemma 2. The following holds for the data of Example 1.

1. For both the private and the public case, the data does not satisfy 2-SARP.

2. For both the private and the public case, the data does satisfy 2-WARP.

Lemma 2 implies the correctness of the following theorem in case $L=2$. As shown in the Appendix, it is possible to construct data sets (similar to the one in Example 1) to obtain exactly the same conclusion in a setting with $L$ household members and $L+1$ goods.

Theorem 1. Let $L \geq 2$. There exists a data set $S$, with only $L+1$ goods, that satisfies $L$-WARP but not $L$-SARP. In general, this implies that $L$-WARP is not equivalent to $L$-SARP for $N+K=L+1$. This non-equivalence conclusion is independent of the public or private nature of the goods.

Labor supply setting. Let us now turn to the restricted labor supply setting. More precisely, we consider a household with $L$ members in which there is only private consumption of the $L+1$ goods. The first $L$ goods represent leisure and are exclusively consumed by individual members. The $(L+1)$-th good is a Hicksian aggregate. We will treat this Hicksian good as a private good for which we do not observe the intrahousehold allocation. As indicated in the Introduction, this restricted setting corresponds to [10]'s labor supply model, which is widely used in empirical analyses of collective consumption behavior. Importantly, while we treat the Hicksian aggregate as a private good to facilitate our discussion (and for 
the analogy with [10]'s original model), our following results actually also hold if the Hicksian aggregate were a public good. ${ }^{8}$

To formally explain the relation between this restricted setting and the general setting that we discussed before, assume a data set $S=\left\{\left(\mathbf{p}_{t}, 0 ; \mathbf{q}_{t}, 0\right), t=1, \ldots, T\right\}$ that contains $L+1$ goods. Then the first $L$ entries of the feasible personalized quantities $\mathbf{q}_{t}^{\ell}$ pertain to the exclusive goods, and are defined as follows for all $t=1, \ldots, T$ and $\ell, j=1, \ldots, L$ :

$$
\left[\mathbf{q}_{t}^{\ell}\right]_{\ell}=\left[\mathbf{q}_{t}\right]_{\ell} \text {, and }\left[\mathbf{q}_{t}^{\ell}\right]_{j}=0 \text { if } \ell \neq j
$$

where we use $[\mathbf{x}]_{i}$ to denote the i-the entry of the vector $\mathbf{x}$. Thus, each member $\ell$ consumes only two goods: the exclusive $\ell$-th good (of which the individual consumption is observed by construction) and a share of the $(L+1)$-th non-exclusive good (of which the individual consumption is not observed). Our proof of Proposition 1 exploits this two-goods feature. In particular, we can build on the original result of [14] to obtain the following conclusion.

Proposition 1. Let $S=\left\{\left(\mathbf{p}_{t}, 0 ; \mathbf{q}_{t}, 0\right), t=1, \ldots, T\right\}$ be a set of observations with $L+1$ goods. Assume households with $L$ members of which each member consumes exclusively one of the goods. Then $L$-WARP is equivalent to L-SARP.

Thus, if $L$ (out of $L+1$ ) goods are exclusive, then transitivity of individual preferences does not have empirical bite. As a result, the empirical analysis of multi-member consumption behavior need not explicitly account for transitivity, which can substantially alleviate the computational burden in practical applications. For instance, [7] introduced an integer programming method to check consistency of a data set $S$ with revealed preference axioms of collective consumption models. ${ }^{9}$ When using this method for the $L$-SARP condition that we consider here, the equivalence result in Proposition 1 implies that we can drop $T^{3}$ transitivity constraints without affecting the conclusions of the analysis. Given that integer programming is often time consuming, this may considerably facilitate the empirical analysis when $T$ gets large.

\section{Conclusion}

We showed that, in general, the equivalence between WARP and SARP for 2 goods does not generalize to $L$-WARP and $L$-SARP for $L+1$ goods. As such, our results describe the settings in which transitivity of the preferences can be meaningfully tested for household/groups with $L$ members that take Pareto optimal decisions. By contrast, the equivalence between $L$-WARP and $L$-SARP does hold for $L+1$ goods if each of the $L$ household members is the exclusive consumer of one good (as in the collective labor supply setting of [10]). In that case, transitivity does not generate empirical bite. This can substantially facilitate the empirical revealed preference analysis in practical applications.

\footnotetext{
${ }^{8}$ The proof of Proposition 1 is easily adapted.

${ }^{9}$ Actually, [7] consider the Generalized Axiom of Revealed Preferences (GARP) rather than SARP in their analysis. However, the integer programming problem for $L$-SARP is directly analogous to the one based on GARP. For compactness, we do not include it here.
} 


\section{References}

[1] S. Afriat. The construction of utility functions from expenditure data. International Economic Review, 8:67-77, 1967.

[2] D. Brown and R. Matzkin. Testable restrictions on the equilibrium manifold. Econometrica, 64:1249-1262, 1996.

[3] C. Camerer, G. Loewenstein, and M. Rabin (Eds.). Advances in Behavioral Economics. Princeton University Press, 2003.

[4] A. Carvajal, I. Ray, and S. Snyder. Equilibrium behavior in markets and games: testable restrictions and identification. Journal of Mathematical Economics, 40:1-40, 2004.

[5] L. Cherchye, B. De Rock, and F. Vermeulen. The collective model of household consumption: a nonparametric characterization. Econometrica, 75:553-574, 2007.

[6] L. Cherchye, B. De Rock, and F. Vermeulen. Collective Household Consumption Behavior: Revealed Preference Analysis. 2010.

[7] L. Cherchye, B. De Rock, and F. Vermeulen. The revealed preference approach to collective consumption behavior: testing and sharing rule recovery. Review of Economic Studies, 78:176-198, 2011.

[8] L. Cherchye, T. Demuynck, and B. De Rock. Testable implications of general equilibrium models: An integer programming approach. Journal of Mathematical Economics, 47:546-575, 2011.

[9] L. Cherchye and B. De Rock V. Platino. Private versus public consumption within groups: testing the nature of goods from aggregate data. Economic Theory, 54:1248$1258,2013$.

[10] P.-A. Chiappori. Rational household labor supply. Econometrica, 56:63-89, 1988.

[11] P.-A. Chiappori and I. Ekeland. The Economics and Mathematics of Aggregation. Foundations and Trends in Microeconomics, 5:1-2. Boston and Delft, 2009.

[12] H. Houthakker. Revealed preference and the utility function. Economics, 17:159-174, 1950.

[13] H. Peters and P. Wakker. WARP does not imply SARP for more than two commodities. Journal of Economic Theory, 62:152-160, 1994.

[14] H. Rose. Consistency of preference: The two-commodity case. Review of Economic Studies, 25:124-125, 1958.

[15] P.A. Samuelson. A note on the pure theory of consumer's behaviour. Economica, 5:61-71, 1938. 
[16] B. Smeulders, L. Cherchye, F.C.R. Spieksma B. De Rock, and F. Talla Nobibon. The weak axiom of revealed preference for collective households. Draft, KU Leuven, Belgium, 2014.

[17] B. Smeulders, L. Cherchye, B. De Rock, and F.C.R. Spieksma. Goodness of fit measures for revealed preference tests: Complexity results and algorithms. ACM Transactions on Economics and Computation, page forthcoming, 2014.

[18] S. Snyder. Observable implications of equilibrium behavior on finite data. Journal of Mathematical Economics, 40:165-176, 2004.

[19] A. Tversky. Intransitivity of preferences. Psychological Review, 76:31-48, 1969.

[20] H. Varian. The nonparametric approach to demand analysis. Econometrica, 50:945-974, 1982.

[21] H. Varian. Revealed preference. Samuelsonian Economics and the 21st Century, 2006.

[22] F. Vermeulen. Collective household models: principles and main results. Journal of Economic Surveys, 16:533-564, 2002.

\section{Appendix}

\section{Proof of Lemma 1}

To show this result, we consider the following specification of feasible personalized quantities and prices. ${ }^{10}$ For all $t=1, \ldots, T, i=1, \ldots, N, j=1, \ldots, L$ :

$$
\begin{gathered}
{\left[\mathbf{P}_{t}^{i}\right]_{j}=0,} \\
{\left[\mathbf{q}_{t}^{i}\right]_{i}=\left[\mathbf{q}_{t}\right]_{i} \text { and }\left[\mathbf{q}_{t}^{i}\right]_{j}=0 \text { if } i \neq j,}
\end{gathered}
$$

i.e. for the first $\mathrm{N}$ members we have that all personalized prices are zero and there is only one non-zero entry for the personalized quantities. For all $t=1, \ldots, T, i=N+1, \ldots, N+K, j=$ $1, \ldots, L$ :

$$
\begin{gathered}
{\left[\mathbf{q}_{t}^{i}\right]_{j}=0,} \\
{\left[\mathbf{P}_{t}^{i}\right]_{i}=\left[\mathbf{P}_{t}\right]_{i} \text { and }\left[\mathbf{P}_{t}^{i}\right]_{j}=0 \text { if } i \neq j,}
\end{gathered}
$$

i.e. for the next $\mathrm{K}$ members all personalized quantities are zero and there is only one nonzero entry for the personalized prices. Finally, if $L>N+K$, for all $t=1, \ldots, T, i=$ $N+K+1, \ldots, L, j=1, \ldots, L$ :

$$
\left[\mathbf{P}_{t}^{i}\right]_{j}=0 \text { and }\left[\mathbf{q}_{t}^{i}\right]_{j}=0,
$$

i.e. for the remaining (N-L-K) members all personalized quantities and personalized prices are zero. Given this specification, one can easily verify that for each $\ell=1, \ldots L$, there is at most one good in the WARP (respectively SARP) test. Our reasoning in the main text then shows that $L$-WARP (respectively $L$-SARP) is a vacuous condition in this setting.

\footnotetext{
${ }^{10}[\mathbf{x}]_{i}$ denotes the $i$-th entry of the vector $\mathbf{x}$.
} 


\section{Proof of Lemma 2}

To prove part 1 of the lemma, we make us of the scalar products in Table 2.

\begin{tabular}{|l|l|l|l|}
\hline $\mathfrak{p}_{1} \mathfrak{q}_{1}=37.5$ & $\mathfrak{p}_{2} \mathfrak{q}_{2}=10$ & $\mathfrak{p}_{3} \mathfrak{q}_{3}=10$ & $\mathfrak{p}_{4} \mathfrak{q}_{4}=11$ \\
& $\mathfrak{p}_{2} \mathfrak{q}_{4}=10.9$ & $\mathfrak{p}_{3} \mathfrak{q}_{2}=10.1$ & $\mathfrak{p}_{4} \mathfrak{q}_{3}=25$ \\
\hline $\mathfrak{p}_{1}\left(\mathfrak{q}_{2}+\mathfrak{q}_{3}+\mathfrak{q}_{4}\right)=37$ & $\mathfrak{p}_{2}\left(\mathfrak{q}_{1}+\mathfrak{q}_{3}\right)=9.5$ & $\mathfrak{p}_{3}\left(\mathfrak{q}_{1}+\mathfrak{q}_{4}\right)=9.86$ & $\mathfrak{p}_{4}\left(\mathfrak{q}_{1}+\mathfrak{q}_{2}\right)=10$ \\
\hline
\end{tabular}

Table 2: Some relevant scalar products.

The numbers of Table 2 show that the following inequalities hold: $\mathfrak{p}_{1} \mathfrak{q}_{1} \geq \mathfrak{p}_{1}\left(\mathfrak{q}_{2}+\mathfrak{q}_{3}+\right.$ $\left.\mathfrak{q}_{4}\right), \mathfrak{p}_{2} \mathfrak{q}_{2} \geq \mathfrak{p}_{2}\left(\mathfrak{q}_{1}+\mathfrak{q}_{3}\right), \mathfrak{p}_{3} \mathfrak{q}_{3} \geq \mathfrak{p}_{3}\left(\mathfrak{q}_{1}+\mathfrak{q}_{4}\right)$, and $\mathfrak{p}_{4} \mathfrak{q}_{4} \geq \mathfrak{p}_{4}\left(\mathfrak{q}_{1}+\mathfrak{q}_{2}\right)$. Assume that all consumption is private and consider any specification of feasible personalized quantities $\mathbf{q}_{t}^{1}$ and $\mathbf{q}_{t}^{2}$. Then, $\mathfrak{p}_{1} \mathfrak{q}_{1} \geq \mathfrak{p}_{1}\left(\mathfrak{q}_{2}+\mathfrak{q}_{3}+\mathfrak{q}_{4}\right)$ implies that there always exists at least one $\ell$ for which $\mathbf{p}_{1} \mathbf{q}_{1}^{\ell} \geq$ $\mathbf{p}_{1}\left(\mathbf{q}_{2}^{\ell}+\mathbf{q}_{3}^{\ell}+\mathbf{q}_{4}^{\ell}\right)$. Indeed, assume this is not the case, i.e. $\mathbf{p}_{1} \mathbf{q}_{1}^{\ell}<\mathbf{p}_{1}\left(\mathbf{q}_{2}^{\ell}+\mathbf{q}_{3}^{\ell}+\mathbf{q}_{4}^{\ell}\right)$ for both $\ell=1$ and $\ell=2$. Adding up these last two inequalities then gives a contradiction: $\mathfrak{p}_{1} \mathfrak{q}_{1}=\mathbf{p}_{1} \mathbf{q}_{1}^{1}+\mathbf{p}_{1} \mathbf{q}_{1}^{2}<\mathbf{p}_{1}\left(\mathbf{q}_{2}^{1}+\mathbf{q}_{3}^{1}+\mathbf{q}_{4}^{1}\right)+\mathbf{p}_{1}\left(\mathbf{q}_{2}^{2}+\mathbf{q}_{3}^{2}+\mathbf{q}_{4}^{2}\right)=\mathfrak{p}_{1}\left(\mathfrak{q}_{2}+\mathfrak{q}_{3}+\mathfrak{q}_{4}\right)$. Without losing generality, let us assume that $\mathbf{p}_{1} \mathbf{q}_{1}^{1} \geq \mathbf{p}_{1}\left(\mathbf{q}_{2}^{1}+\mathbf{q}_{3}^{1}+\mathbf{q}_{4}^{1}\right)$.

A similar reasoning applies to $\mathfrak{p}_{2} \mathfrak{q}_{2} \geq \mathfrak{p}_{2}\left(\mathfrak{q}_{1}+\mathfrak{q}_{3}\right)$ and $\mathfrak{p}_{3} \mathfrak{q}_{3} \geq \mathfrak{p}_{3}\left(\mathfrak{q}_{1}+\mathfrak{q}_{4}\right)$. However, since $\mathbf{p}_{1} \mathbf{q}_{1}^{1} \geq \mathbf{p}_{1}\left(\mathbf{q}_{2}^{1}+\mathbf{q}_{3}^{1}+\mathbf{q}_{4}^{1}\right)$, we can now conclude that it must be that $\mathbf{p}_{2} \mathbf{q}_{2}^{2} \geq \mathbf{p}_{2}\left(\mathbf{q}_{1}^{2}+\mathbf{q}_{3}^{2}\right)$ and $\mathbf{p}_{3} \mathbf{q}_{3}^{2} \geq \mathbf{p}_{3}\left(\mathbf{q}_{1}^{2}+\mathbf{q}_{4}^{2}\right)$. Indeed, otherwise we would have feasible personalized quantities that lead to a SARP rejection for $\ell=1$. Note that these inequalities imply that $2 R_{0}^{2} 3$ and $3 R_{0}^{2} 4$, meaning that $2 R^{2} 4$.

Finally, using the same argument once more for $\mathfrak{p}_{4} \mathfrak{q}_{4} \geq \mathfrak{p}_{4}\left(\mathfrak{q}_{1}+\mathfrak{q}_{2}\right)$, we can conclude that any specification of feasible personalized quantities leads to a rejection of 2 -SARP. We always obtain a rejection of SARP for either $\ell=1$ or $\ell=2$. To finish the proof of part 1 , we have to note that exactly the same reasoning holds if all goods are public (or even any intermediate case with both private and public goods).

To prove part 2 of the lemma, we need to give one specification of feasible personalized quantities that satisfies 2-WARP, and another specification of feasible personalized prices that satisfies 2-WARP.

For the private case, let us consider the specification of feasible personalized quantities in Table 3.

$$
\begin{array}{|l|l|}
\hline \mathbf{q}_{1}^{1}=(5,0,0) & \mathbf{q}_{1}^{2}=(0,0,0) \\
\mathbf{q}_{2}^{1}=(0,0,0) & \mathbf{q}_{2}^{2}=(0,5,0) \\
\mathbf{q}_{3}^{1}=(0,0,0) & \mathbf{q}_{3}^{2}=(0,0,5) \\
\mathbf{q}_{4}^{1}=(0,0,0) & \mathbf{q}_{4}^{2}=(4,3,1) \\
\hline
\end{array}
$$

Table 3: Values of $\mathbf{q}_{t}^{1}$ and $\mathbf{q}_{t}^{2}$ for $t=1,2,3,4$.

For this specification we obtain the scalar products in Table 4, which allow us to conclude that 2-WARP is satisfied.

Similarly, for the public case, we consider the specification of feasible personalized prices in Table 5. 


\begin{tabular}{|l|l|l|l|}
\hline $\mathbf{p}_{1} \mathbf{q}_{1}^{1}=37.5$ & $\mathbf{p}_{2} \mathbf{q}_{1}^{1}=5$ & $\mathbf{p}_{3} \mathbf{q}_{1}^{1}=1$ & $\mathbf{p}_{4} \mathbf{q}_{1}^{1}=5$ \\
$\mathbf{p}_{1} \mathbf{q}_{2}^{1}=0$ & $\mathbf{p}_{2} \mathbf{q}_{2}^{1}=0$ & $\mathbf{p}_{3} \mathbf{q}_{2}^{1}=0$ & $\mathbf{p}_{4} \mathbf{q}_{2}^{1}=0$ \\
$\mathbf{p}_{1} \mathbf{q}_{3}^{1}=0$ & $\mathbf{p}_{2} \mathbf{q}_{3}^{1}=0$ & $\mathbf{p}_{3} \mathbf{q}_{3}^{1}=0$ & $\mathbf{p}_{4} \mathbf{q}_{3}^{1}=0$ \\
$\mathbf{p}_{1} \mathbf{q}_{4}^{1}=0$ & $\mathbf{p}_{2} \mathbf{q}_{4}^{1}=0$ & $\mathbf{p}_{3} \mathbf{q}_{4}^{1}=0$ & $\mathbf{p}_{4} \mathbf{q}_{4}^{1}=0$ \\
\hline $\mathbf{p}_{1} \mathbf{q}_{1}^{2}=0$ & $\mathbf{p}_{2} \mathbf{q}_{1}^{2}=0$ & $\mathbf{p}_{3} \mathbf{q}_{1}^{2}=0$ & $\mathbf{p}_{4} \mathbf{q}_{1}^{2}=0$ \\
$\mathbf{p}_{1} \mathbf{q}_{2}^{2}=2.5$ & $\mathbf{p}_{2} \mathbf{q}_{2}^{2}=10$ & $\mathbf{p}_{3} \mathbf{q}_{2}^{2}=10.1$ & $\mathbf{p}_{4} \mathbf{q}_{2}^{2}=5$ \\
$\mathbf{p}_{1} \mathbf{q}_{3}^{2}=2.5$ & $\mathbf{p}_{2} \mathbf{q}_{3}^{2}=4.5$ & $\mathbf{p}_{3} \mathbf{q}_{3}^{2}=10$ & $\mathbf{p}_{4} \mathbf{q}_{3}^{2}=20$ \\
$\mathbf{p}_{1} \mathbf{q}_{4}^{2}=32$ & $\mathbf{p}_{2} \mathbf{q}_{4}^{2}=10.9$ & $\mathbf{p}_{3} \mathbf{q}_{4}^{2}=8.86$ & $\mathbf{p}_{4} \mathbf{q}_{4}^{2}=11$ \\
\hline
\end{tabular}

Table 4: Scalar products for the private case.

\begin{tabular}{|l|l|}
\hline $\mathbf{P}_{1}^{1}=(7.5,0,0)$ & $\mathbf{P}_{1}^{2}=(0,0.5,0.5)$ \\
$\mathbf{P}_{2}^{1}=(0.2,0.1,0.2)$ & $\mathbf{P}_{2}^{2}=(0.8,1.9,0.7)$ \\
$\mathbf{P}_{3}^{1}=(0.2,0.1,0.1)$ & $\mathbf{P}_{3}^{2}=(0,1.92,1.9)$ \\
$\mathbf{P}_{4}^{1}=(1,0,0)$ & $\mathbf{P}_{4}^{2}=(0,1,5)$ \\
\hline
\end{tabular}

Table 5: Values of $\mathbf{P}_{t}^{1}$ and $\mathbf{P}_{t}^{2}$ for $t=1,2,3,4$.

For this specification we obtain the scalar products in Table 6 , which allow us to conclude that 2-WARP is satisfied.

\begin{tabular}{|l|l|l|l|}
\hline $\mathbf{P}_{1}^{1} \mathbf{Q}_{1}=37.5$ & $\mathbf{P}_{2}^{1} \mathbf{Q}_{1}=1$ & $\mathbf{P}_{3}^{1} \mathbf{Q}_{1}=1$ & $\mathbf{P}_{4}^{1} \mathbf{Q}_{1}=5$ \\
$\mathbf{P}_{1}^{1} \mathbf{Q}_{2}=0$ & $\mathbf{P}_{2}^{1} \mathbf{Q}_{2}=0.5$ & $\mathbf{P}_{3}^{1} \mathbf{Q}_{2}=0.5$ & $\mathbf{P}_{4}^{1} \mathbf{Q}_{2}=0$ \\
$\mathbf{P}_{1}^{1} \mathbf{Q}_{3}=0$ & $\mathbf{P}_{2}^{1} \mathbf{Q}_{3}=1$ & $\mathbf{P}_{3}^{1} \mathbf{Q}_{3}=0.5$ & $\mathbf{P}_{4}^{1} \mathbf{Q}_{3}=0$ \\
$\mathbf{P}_{1}^{1} \mathbf{Q}_{4}=30$ & $\mathbf{P}_{2}^{1} \mathbf{Q}_{4}=1.3$ & $\mathbf{P}_{3}^{1} \mathbf{Q}_{4}=1.2$ & $\mathbf{P}_{4}^{1} Q_{4}=4$ \\
\hline $\mathbf{P}_{1}^{2} \mathbf{Q}_{1}=0$ & $\mathbf{P}_{2}^{2} \mathbf{Q}_{1}=4$ & $\mathbf{P}_{3}^{2} \mathbf{Q}_{1}=0$ & $\mathbf{P}_{4}^{2} \mathbf{Q}_{1}=0$ \\
$\mathbf{P}_{1}^{2} \mathbf{Q}_{2}=2.5$ & $\mathbf{P}_{2}^{2} \mathbf{Q}_{2}=9.5$ & $\mathbf{P}_{3}^{2} \mathbf{Q}_{2}=9.6$ & $\mathbf{P}_{4}^{2} \mathbf{Q}_{2}=5$ \\
$\mathbf{P}_{1}^{2} \mathbf{Q}_{3}=2.5$ & $\mathbf{P}_{2}^{2} \mathbf{Q}_{3}=3.5$ & $\mathbf{P}_{3}^{2} \mathbf{Q}_{3}=9.5$ & $\mathbf{P}_{4}^{2} \mathbf{Q}_{3}=25$ \\
$\mathbf{P}_{1}^{2} \mathbf{Q}_{4}=2$ & $\mathbf{P}_{2}^{2} \mathbf{Q}_{4}=9.6$ & $\mathbf{P}_{3}^{2} \mathbf{Q}_{4}=7.66$ & $\mathbf{P}_{4}^{2} \mathbf{Q}_{4}=8$ \\
\hline
\end{tabular}

Table 6: Scalar products for the public case.

\section{Proof of Theorem 1}

Take $L \geq 3$. We start with the dataset $S$ from example 1 and we add goods $4,5, \ldots, L+1$ and observations $5,6, \ldots, L+2$ to it. The quantities for goods $4,5, \ldots, L+1$ are 0 in observations $1,2,3$ and 4 and the corresponding prices in these observations are $\epsilon<\frac{0.14}{L}$. It can be checked that in this case the following inequalities hold: $\mathfrak{p}_{1} \mathfrak{q}_{1}>\mathfrak{p}_{1}\left(\mathfrak{q}_{2}+\ldots+\mathfrak{q}_{L+2}\right), \mathfrak{p}_{2} \mathfrak{q}_{2}>\mathfrak{p}_{2}\left(\mathfrak{q}_{1}+\right.$ $\left.\mathfrak{q}_{3}+\mathfrak{q}_{5}+\ldots+\mathfrak{q}_{L+2}\right), \mathfrak{p}_{3} \mathfrak{q}_{3}>\mathfrak{p}_{3}\left(\mathfrak{q}_{1}+\mathfrak{q}_{4}+\mathfrak{q}_{5}+\ldots+\mathfrak{q}_{L+2}\right)$ and $\mathfrak{p}_{4} \mathfrak{q}_{4}>\mathfrak{p}_{4}\left(\mathfrak{q}_{1}+\mathfrak{q}_{2}+\mathfrak{q}_{5}+\ldots+\mathfrak{q}_{L+2}\right)$.

For each observation $t=5,6, \ldots, L+2$, it is the case that $\mathfrak{q}_{t}=(0, \ldots, 0,1,0, \ldots, 0)$, with the non-zero quantity for the $(t-1)^{t h}$ good. The corresponding prices are $\mathfrak{p}_{t}=$ $(\epsilon, \ldots, \epsilon, 1, \epsilon, \ldots, \epsilon)$. Again, it can be checked that $\mathfrak{p}_{t} \mathfrak{q}_{t}>\mathfrak{p}_{t}\left(\mathfrak{q}_{1}+\ldots+\mathfrak{q}_{t-1}+\mathfrak{q}_{t+1}+\mathfrak{q}_{L+2}\right)$.

A similar reasoning as in Lemma 2 then shows that this dataset does not satisfy $L$ SARP. Personalized quantities or prices can be found as follows. Assign all goods (prices) of observations 1,2,3 and 4 to members 1 and 2 as in the original examples. Furthermore, assign all goods and prices) of observation $k \geq 5$ to member $k-2$ (with $k=5, \ldots, L+2$ ). 


\section{Proof of Proposition 1}

Clearly, if $S$ is a data set that satisfies $L$-SARP, then it also needs to satisfy $L$-WARP. So we only need to prove the reverse statement. Let $S$ be a data set that satisfies $L$-WARP. This means that there exist feasible personalized quantities $\mathbf{q}_{t}^{\ell}$ such that for each $\ell=1, \ldots, L$ the data $\left\{\left(\mathbf{p}_{t}, \mathbf{q}_{t}^{\ell}\right), t=, 1, \ldots, T\right\}$ satisfies WARP. By construction, all entries of $\mathbf{q}_{t}^{\ell}$ are zero except for the $\ell$-th and $L+1$-th entries. Clearly, all the zero entries are irrelevant for checking consistency with WARP. Therefore, we can use [14]'s result to conclude that, for each member $\ell$, the corresponding SARP condition is met, and thus that $S$ satisfies $L$-SARP. 